\title{
Subclinical thyroid disease: consensus or conundrum?
}

\section{Editorial}

Subclinical thyroid dysfunction is defined as an abnormal serum TSH level (reference range: 0.45 to $4.50 \mu \mathrm{U}$ per $\mathrm{mL}$ ) and free T4and $\mathrm{T} 3$ levels within their reference ranges.

Thyroid disease is common and may present to a wide range of health professionals in clinical practice. With the advent of TSH radioimmunoassay in the 70 s, the entity of mildly elevated TSH and normal serum thyroid hormones levels was increasingly recognized. Afterwards the introduction of the second and third generation sensitive TSH in the 80s identified the entity of subclinical hyperthyroidism in which serum TSH is suppressed and serum T4 and T3 levels are normal.

The management of subclinical thyroid dysfunction is controversial. The prevalence of subclinical hypothyroidism is about 4 to 8.5 percent, and may be as high as 20 percent in women older than 60 years. Subclinical hyperthyroidism is found in approximately $2 \%$ of the population.

STD and hypothyroid disease are laboratory diagnoses and there is good evidence that subclinical hypothyroidism is associated with progression to overt disease especially when it is associated with positive TPO, yet most well reputed institutions recommend against routine screening of asymptomatic patients, but screening is recommended for high-risk populations.

In 2002, a scientific review and consensus committee, which included representatives from the American Thyroid Association, the American Association of Clinical Endocrinologists, and the Endocrine Society convened a panel of expertise to define STD and review the literatures regarding the risk and benefit of treatment, the panel had come out with definition of subclinical hypothyroidism as (a serum TSH concentration above the statistically defined upper limit of the reference range when serum free $\mathrm{T} 4$ is within its reference range, and subclinical hyperthyroidism; a serum TSH concentration below the statistically defined lower limit of the reference range when serum FT4 and T3 levels are within their reference ranges.)

Despite a working definition of STD, the panel found little evidence to guide physicians in managing subclinical hyperthyroidism and hypothyroidism. Some patients will progress to overt disease, and in some patients, the serum TSH concentration will remain stable over time or will spontaneously return to the reference range. There also is controversy regarding what, if any, adverse outcomes occur from subclinical thyroid disease, and whether benefit can be expected from treatment. As a result, various organizations have adopted diverse recommendations regarding screening for STD.

Subclinical hyperthyroidism and subclinical hypothyroidism are common clinical entities in current practice. The diagnosis of subclinical thyroid disease is based on the sensitivity of the hypothalamic-pituitary-thyroid axis, therefore it has been an almost

\author{
Volume 4 Issue 4 - 2017
}

\author{
Saadi AlJadir \\ Department of Endocrinology, Gulf Medical University, UAE
}

Correspondence: Saadi AlJadir, Department of Endocrinology, Gulf Medical University, P.O. Box 3243, Fujairah, UAE, Email saljadir5।@gmail.com

Received: April 20, 2017| Published: April 21, 2017

biochemical diagnosis more than been a clinical one! In unselected populations, measurement of serum TSH has a sensitivity of $89-95 \%$ and a specificity of $90-96 \%$ for overt thyroid dysfunction, compared with cases confirmed by history, examination and additional testing. Normal serum TSH concentrations are found in some patients with hypothyroidism caused by pituitary or hypothalamic disease, although both these situations are rarely encountered in routine clinical practice.

There is good evidence that subclinical hypothyroidism is associated with progression to overt disease. Patients with a serum TSH level greater than $10 \mu \mathrm{U}$ per $\mathrm{mL}$ have a higher incidence of elevated serum LDL cholesterol concentrations; however, evidence is lacking for other associations. There is insufficient evidence that treatment of subclinical hypothyroidism is beneficial except probably in pregnancy. A serum TSH level of less than $0.1 \mu \mathrm{U}$ per $\mathrm{mL}$ is associated with progression to overt hyperthyroidism, atrial fibrillation, reduced bone mineral density, and cardiac dysfunction. There is little evidence that early treatment alters the clinical course.

During pregnancy the diagnosis of SCT mandates understanding of changes of thyroid physiology that are reflected in altered thyroid function tests. The major changes in thyroid function during pregnancy are due to an increase in serum TBG concentrations and stimulation of the TSH receptor by hCG. The use of trimester-specific reference ranges for serum TSH and method and trimester-specific reference ranges for serum free $\mathrm{T} 4$ are now recommended. The reference range for TSH levels is lower particularly in the first trimester of pregnancy. At 2009 Endocrine society had adopted new recommendations for TSH levels during pregnancy: First trimester: less than 2.5 with a range of $0.1-2.5$ and $(0.2-3.0),(0.3-30)$ for $2^{\text {nd }}$ and $3^{\text {rd }}$ trimesters respectively. If the TSH is greater than 2.5 at any time during pregnancy, T4 levels should be checked to determine whether the hypothyroidism is overt or subclinical. The effect of subclinical hypothyroidism on fetal neurocognitive development is not clear, but one large study showed lower IQ tests in the children of untreated women. In a small percentage of women's TSH can be much suppressed $(<0.01 \mathrm{mIU} / \mathrm{L})$, yet still represent a normal pregnancy. There are slight but significant ethnic differences in TSH levels. 
The findings of slightly elevated TSH and normal thyroid hormone levels do not necessarily imply the presence of subclinical hypothyroidism. Several medications and conditions are known to cause an elevation in TSH. Some drugs such as sulfonylureas, lithium, amiodarone, and iodine..., other conditions that causes elevated TSH include thyroid hormone resistance psychiatric illness, adrenal insufficiency, renal failure, hyperprolactinemia and systemic illness (NTI).

In the Whickham survey, TSH levels above $6 \mathrm{mIU} / 1$ were approximately three times more common in females $(7.5 \%)$ than in males $(2.8 \%)$ and occurred more frequently in females over 45 years of age. TSH levels also showed a progressive increase with age in women but not in men. The prevalence of subclinical hypothyroidism varies from $4.3-9 \%$ there is also a strong association between positive antithyroid antibodies and elevated TSH (TPO and Not TG Abs!)

After 20 years of follow-up of subjects in the Whickham Survey, the risk of overt hypothyroidism was found to be $4.3 \%$ per year in women with elevated TSH and antithyroid antibodies at baseline. The possible advantage of treating subclinical hypothyroidism generally prevents the progression to overt hypothyroidism. Thyroxine therapy may improve the serum lipid profile and which might potentially decrease the risk cardiovascular disease, also treatment may reverse the symptoms of mild hypothyroidism, including psychiatric and cognitive abnormalities.

Subclinical hyperthyroidism is defined as a persistently suppressed serum TSH with normal T4 and T3 in patients who do not have symptoms. Subclinical hyperthyroidism can be caused by the same thyroid disorders that result in clinical hyperthyroidism. Suppressed TSH levels may occasionally result from non-thyroidal causes. The most common cause of subclinical hyperthyroidism is excessive thyroid hormone therapy. Prevalence of subclinical hyperthyroidism was reported to be $10 \%$ in Whickham Survey, and $12 \%$ in the Framingham study, more recently, the Colorado Thyroid Disease Prevalence Study involving 25,862 subjects showed a prevalence of $2.1 \%$. In the Whickham Survey where 2,779 subjects were followed up for 20 years, the incidence of hyperthyroidism in the surviving women was $3.9 \%$ and the calculated incidence rate was only $0.8 / 1,000 /$ year.

The initial TSH level also appears to influence outcome. Subclinical hyperthyroid subjects with initial suppressed but detectable TSH levels tend to return to normal on follow-up while those with undetectable TSH remained unchanged with a small risk of progression to frank hyperthyroidism. The rate of progression to overt hyperthyroidism has been estimated to be $5 \%$ per year with subjects with autonomous thyroid adenoma and nodular goiter. Therefore, the likelihood of progression of subclinical hyperthyroidism to overt disease is small.

Subclinical hyperthyroidism is associated with an increased risk of atrial fibrillation in older adults, and with decreased bone mineral density in postmenopausal women; however, the effectiveness of treatment in preventing these conditions is unknown.

There is lesser-quality evidence suggesting an association between subclinical hyperthyroidism and other cardiovascular effects, including increased heart rate and left ventricular mass, and increased bone turnover markers. Possible associations between subclinical hyperthyroidism and quality of life's parameters, cognition, and increased mortality rates are controversial.

\section{Acknowledgements}

None.

\section{Conflicts of interest}

The author declares there is no conflict of interest. 